Research Article

\title{
The Role of Oxidative Stress in Decreased Acetylcholinesterase Activity at the Neuromuscular Junction of the Diaphragm during Sepsis
}

\author{
Hua Liu, ${ }^{1,2}$ Jin Wu, ${ }^{1}$ Jun-yan Yao, ${ }^{1}$ Hong Wang, ${ }^{1}$ and Shi-tong $\mathrm{Li}^{1}$ \\ ${ }^{1}$ Department of Anesthesiology, Shanghai General Hospital, Shanghai Jiao Tong University School of Medicine, \\ Shanghai 200080, China \\ ${ }^{2}$ Department of Anesthesiology, The Ninth People's Hospital, Shanghai Jiao Tong University School of Medicine, \\ Shanghai 200011, China
}

Correspondence should be addressed to Shi-tong Li; lishitongs@yahoo.com

Received 13 July 2017; Accepted 17 October 2017; Published 5 November 2017

Academic Editor: Paola Venditti

Copyright ( 92017 Hua Liu et al. This is an open access article distributed under the Creative Commons Attribution License, which permits unrestricted use, distribution, and reproduction in any medium, provided the original work is properly cited.

Our recent study demonstrated that acetylcholinesterase (AChE) activity at the neuromuscular junction (NMJ) of the diaphragm decreased during sepsis. However, the mechanisms were not clearly identified. In this study, we aimed to investigate whether the decreased AChE activity was related to oxidative stress by observing AChE activity in different grades of sepsis induced by caecal ligation and puncture (CLP). At $24 \mathrm{~h}$ after surgery, an assay of thiobarbituric acid reactive species (TBARS) and protein carbonyls, as well as the myeloperoxidase (MPO), superoxide dismutase (SOD), and catalase (CAT) activity, was conducted. AChE activity was measured by biochemical and histological detection. AChE and CAT activity in the diaphragm decreased, while the contents of TBARS and protein carbonyls, the activity of MPO and SOD, and the SOD/CAT ratios increased. The above changes were much more significant in the mid-grade septic group than in the low-grade septic group. The colour of the AChE activity staining at the NMJ gradually lightened from the sham surgery group to the mid-grade septic group. AChE activity was significantly negatively correlated with the levels of TBARS and protein carbonyls. We consider that oxidative stress might be responsible for decreased AChE activity in the diaphragms of rats induced with sepsis.

\section{Introduction}

Sepsis is a clinical syndrome caused by severe infection, leading to multiple organ dysfunction $[1,2]$. Our recent study has reported that acetylcholinesterase (AChE) activity at the neuromuscular junction (NMJ) of the diaphragm was inhibited during sepsis [3]. However, the effects of different grades of sepsis on the activity of AChE were not illustrated in that study. Moreover, the mechanisms through which AChE activity was inhibited during sepsis were not clearly identified.

Previous studies have demonstrated that several factors, including inflammatory, immune, hormonal, metabolic, and bioenergetic responses, were involved in the pathogenesis of sepsis [4-6]. One of the crucial factors in these processes is the loss of balance between the reactive species (ROS) and antioxidant systems, leading to a state of irreversible oxidative stress [7]. ROS contain a series of active oxygen free radical or molecule, including superoxide $\left(\mathrm{O}_{2}{ }^{--}\right)$, hydrogen peroxide $\left(\mathrm{H}_{2} \mathrm{O}_{2}\right)$, hydroxyl radicals $\left({ }^{\circ} \mathrm{OH}\right)$, and hypochlorous acid $(\mathrm{HOCl})[8,9]$, which can attack proteins, carbohydrates, nucleic acids, and unsaturated lipids, subsequently causing tissue and organism injury [10]. Several previous studies have found that ROS can inhibit AChE in various tissues [11-17].

These findings raise the possibility that oxidative stress might be a vital contributor to the inhibition of AChE activity during sepsis. In the current study, we detected AChE activity 
in two different grades of sepsis to more intensively determine the effects of sepsis on AChE activity. Meanwhile, we measured the indicators of oxidative injury (thiobarbituric acid reactive species (TBARS) and protein carbonyl) to observe the correlation between AChE activity and oxidative stress during sepsis. We aimed to clarify the role of oxidative stress in the decreased AChE activity at the NMJ of diaphragm during sepsis.

\section{Materials and Methods}

2.1. Animals and Experimental Protocol. Our study was approved by the Animal Care and Use Committee of Shanghai General Hospital Affiliated to Shanghai Jiao Tong University (Grant number 2017DW001). Experiments were performed using a total of 28 adult male Sprague-Dawley rats weighing between 220 and $260 \mathrm{~g}$. The animals were allowed unrestrained access to food and water and housed at an ambient temperature of $23-25^{\circ} \mathrm{C}$ with $12 \mathrm{~h}$ light-dark cycles throughout the study. The rats were randomly divided into three groups: (1) the sham group (S group, $n=8$ ), (2) the low-grade sepsis group (L group, $n=8$ ), and (3) the midgrade sepsis group (M group, $n=12$ due to an expected mortality rate of approximately $25 \%$ within the first $24 \mathrm{~h}$ in our previous study). The rats were anaesthetized with intraperitoneal injection of pentobarbital ( $50 \mathrm{mg} / \mathrm{kg}$ ). In the $\mathrm{L}$ and $\mathrm{M}$ groups, sepsis was surgically induced using the CLP method, as it is widely used and known to closely mimic the pathophysiology of septic patients [18]. Under aseptic conditions, a $3 \mathrm{~cm}$ midline abdominal incision was performed to expose the caecum, with adjoining intestine. The caecum in the $\mathrm{L}$ group was ligated near the distal pole comprising $10 \%$ of the caecum, whereas the caecum in the $M$ group was ligated in the middle portion [19]. Then, the caecum was perforated once with an 18-gauge needle. Faecal droplets were squeezed out of the caecum through penetration holes. The caecum was then relocated into the abdominal cavity, and the laparotomy was closed with 3-0 silk sutures. In the $S$ group, a midline abdominal incision was made, and the caecum was exposed but not ligated or perforated. All rats were immediately resuscitated with prewarmed $\left(37^{\circ} \mathrm{C}\right)$ normal saline (50 ml/kg subcutaneous). All animals were allowed to return to their cages, with free access to food and water after surgery.

At $24 \mathrm{~h}$ after surgery, the surviving rats were euthanized through intraperitoneal injection of pentobarbital (100 mg/kg), and their diaphragms were harvested. The right hemidiaphragm was removed and immediately stored at $-80^{\circ} \mathrm{C}$ until being assayed for AChE activity, as well as the contents of TBARS formation and protein carbonyls, and MPO, SOD, and CAT activities. The ventral costal region of the left hemidiaphragm was immediately removed for histological detection of AChE activity at the NMJ.

2.2. Histological Detection of AChE Activity at the NMJ. The AChE activity at the NMJ of the diaphragm was detected through a modified Karnovsky and Roots method by referencing our previous study [3]. The brown insoluble products on the muscle fibres indicated AChE activity [20].
2.3. Biochemical Measurement of AChE Activity. The method is based on the fact that AChE can hydrolyse acetylcholine to form acetic acid and choline. The latter can react with a mercapto colour reagent to form a yellow symmetrical trinitrobenzene compound. Measuring the colour depth of the compound can reflect the activity of AChE. The AChE activity assay test was carried out using an AChE assay kit from the Nanjing Jiancheng Bioengineering Institute (A024, Nanjing, China) according to the manufacturer's instructions [21]. The results were determined by measuring the absorbance at $412 \mathrm{~nm}$ and expressed as units of AChE activity per milligram of protein.

2.4. TBARS Measurement. As an indicator of lipid peroxidation in oxidative stress, we determined the formation of TBARS during an acid-heating reaction, as previously described [22]. Briefly, the samples were mixed with $1 \mathrm{ml}$ of trichloroacetic acid 10\% purchased from Sigma Chemical (St. Louis, MO, USA) and $1 \mathrm{ml}$ thiobarbituric acid $0.67 \%$, and then, the mixture was heated in a boiling water bath for 15 mins. TBARS was determined by measuring the absorbance at $535 \mathrm{~nm}$, and the results are expressed as malondialdehyde (MDA) equivalents per milligram of protein.

2.5. Measurement of Protein Carbonyls. As a marker of the oxidative damage to proteins, protein carbonyl groups were measured based on the reaction with dinitrophenyl hydrazine, as previously described [23]. Briefly, the proteins were precipitated by adding $20 \%$ trichloroacetic acid and redissolved in dinitrophenyl hydrazine. Eventually, the protein carbonyls were determined by reading the absorbance at $375 \mathrm{~nm}$, and the results were expressed as nmols of protein carbonyls per milligram of protein.

2.6. MPO Activity Assay. MPO activity in homogenates of diaphragm tissue was determined using a test kit from Nanjing Jiancheng Bioengineering Institute (A044, Nanjing, China). Activity was measured spectrophotometrically as the change in absorbance at $412 \mathrm{~nm}$, using a Spectramax microplate reader. The results are expressed as units of MPO activity per gram of tissue wet weight.

2.7. Measurement of CAT and SOD Activities. The aboveprepared supernatant was used for CAT and SOD activity assay according to the instructions of their corresponding test kit purchased from Nanjing Jiancheng Bioengineering Institute (CAT: A007-1; SOD: A001-1, Nanjing, China). Both results were expressed as units of enzyme activity per gram of tissue wet weight. Finally, the ratio of SOD/CAT was calculated and recorded.

2.8. Statistical Analyses. SPSS (version 19.0; SPSS Inc., Chicago, Illinois, USA) was used for the statistical analysis. Values are expressed as the mean \pm standard deviation (SD). The data were tested for normality and equality of variance. Between-group comparisons for each dependent variable were assessed using analysis of variance (ANOVA) with the least significant difference (LSD) test. Pearson's correlation analysis was used to determine the association 


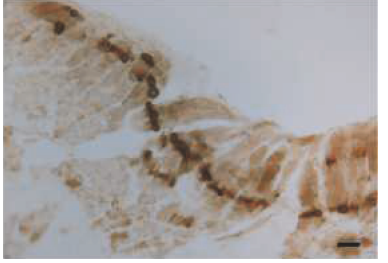

(a)

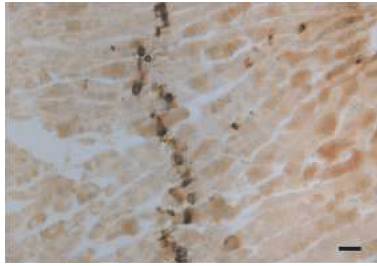

(b)

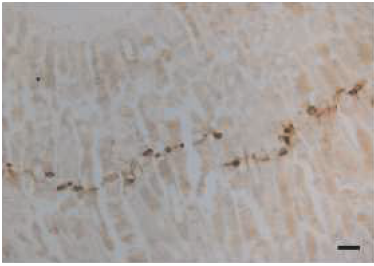

(c)

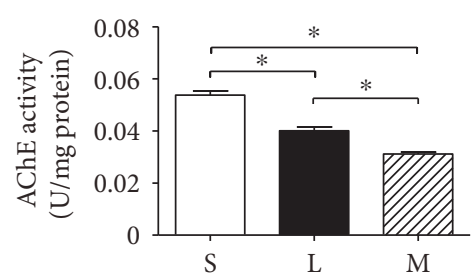

(d)

Figure 1: AChE staining at neuromuscular junction in sham group (a), low-grade septic group (b), and midgrade septic group (c). Bars $=50 \mu \mathrm{m}$. Comparison of AChE activity through biochemical measurement in three groups of rat diaphragm (d). The values are expressed as means \pm SD. $n=8,{ }^{*} P<0.01$. S: sham group; L: low-grade septic group; M: midgrade septic group; SD: standard deviation.

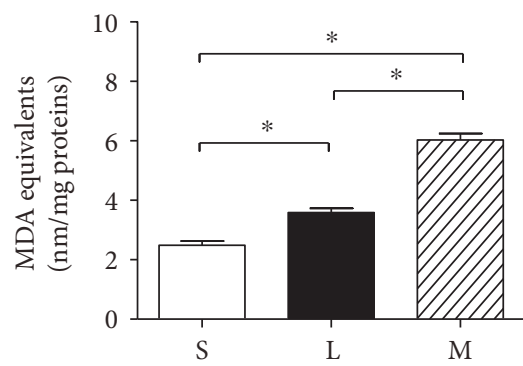

(a)

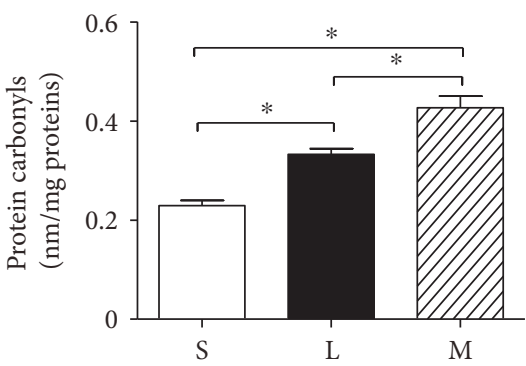

(b)

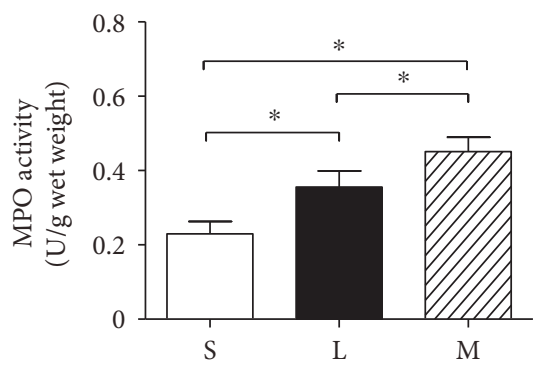

(c)

FIgURE 2: Comparison of thiobarbituric acid reactive species (a), protein carbonyls (b), and MPO activity (c) in three groups of rat diaphragm. The values are expressed as means \pm SD. $n=8,{ }^{*} P<0.01$. S: sham group; L: low-grade septic group; M: midgrade septic group; SD: standard deviation.

between AChE activity and TBARS or protein carbonyls, and $P<0.05$ was considered to be statistically significant.

\section{Results}

3.1. Mortality within $24 \mathrm{~h}$. Four of the 12 rats in the $\mathrm{M}$ group died within $24 \mathrm{~h}$, and all other rats survived during the first $24 \mathrm{~h}$. In addition, the rats in midgrade septic group developed more severe septic symptoms, such as shortness of breath, hair erection, subconjunctival haemorrhage, diarrhoea, and loss of movement, than those in low-grade septic group.

3.2. AChE Activity Staining at the NMJ. As shown in Figure 1, AChE staining was detected at motor endplate regions in rat diaphragms from all three groups. Observed from the slices, the brown insoluble products of AChE staining in the $\mathrm{S}$ group were deepest, while the brown in $\mathrm{M}$ group was lightest (Figures 1(a), 1(b), and 1(c)).

3.3. AChE Activity in the Diaphragm. Compared with the $\mathrm{S}$ group, AChE activity decreased significantly in both the $\mathrm{L}$ and $\mathrm{M}$ groups $(P<0.01)$. Furthermore, the decline of AChE activity in the $M$ group was much more significant than that in the L group $(P<0.01)$ (Figure $1(\mathrm{~d})$ ).

\subsection{Oxidative Parameters at the Diaphragm}

3.4.1. TBARS and Protein Carbonyls in the Diaphragm. Twenty-four hours after CLP (in both the L and M groups), TBARS and protein carbonyls increased significantly in the diaphragm $(P<0.01)$. Additionally, the levels of TBARS and protein carbonyls were higher in the $\mathrm{M}$ group than those in the $\mathrm{L}$ group $(P<0.01)$ (Figures $2(\mathrm{a})$ and $2(\mathrm{~b}))$.

3.4.2. MPO Activity in the Diaphragm. As illustrated in Figure 2(c), MPO activity was significantly elevated in rats in both the $\mathrm{L}$ and $\mathrm{M}$ groups compared to rats in the $\mathrm{S}$ group $(P<0.01)$. Moreover, MPO activity was higher in the $\mathrm{M}$ group than that in the L group $(P<0.01)$ (Figure $2(\mathrm{c})$ ).

\subsection{Antioxidant Enzyme Activities}

3.5.1. SOD and CAT Activities and SOD/CAT Ratios. SOD activity increased in both the $\mathrm{L}$ and $\mathrm{M}$ groups $(P<0.01)$, and the increase of SOD activity was higher in the M group than that in the $\mathrm{L}$ group (Figure 3(a)). In contrast, a significant decrease was observed in CAT activity in the $\mathrm{L}$ $(P<0.05)$ and $\mathrm{M}$ groups $(P<0.01)$. CAT activity decreased more in the $\mathrm{M}$ group than in the $\mathrm{L}$ group (Figure $3(\mathrm{~b})$ ). The ratio of SOD and CAT increased significantly in the L group $(P<0.05)$ and $\mathrm{M}$ group $(P<0.01)$. Meanwhile, this ratio was higher in the $\mathrm{M}$ group than that in the $\mathrm{L}$ group $(P<0.01)$ (Figure 3(c)).

3.6. Correlation between AChE Activity and TBARS or Protein Carbonyls. AChE activity was significantly and negatively correlated with the levels of TBARS $(r=-0.839$, $P<0.01)$ (Figure $4(\mathrm{a}))$ and protein carbonyls $(r=-0.857$, $P<0.01$ ) (Figure 4(b)). 


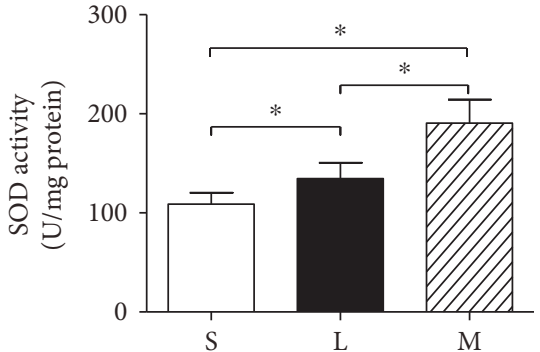

(a)

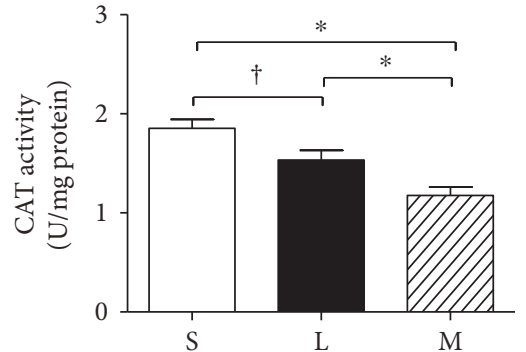

(b)

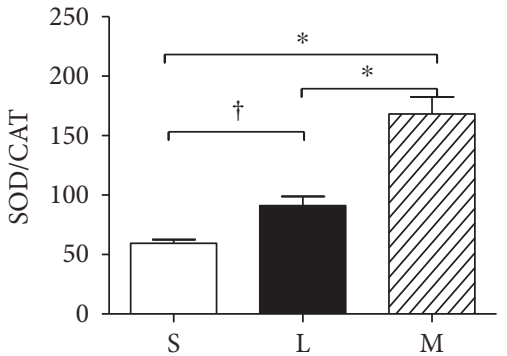

(c)

FIGURE 3: Comparison of SOD activity (a), CAT activity (b), and SOD/CAT (c) in three groups of rat diaphragms. The values are expressed as the means \pm SD. $n=8,{ }^{*} P<0.01,{ }^{\dagger} P<0.05$. S: sham group; L: low-grade septic group; M: midgrade septic group; SD: standard deviation.

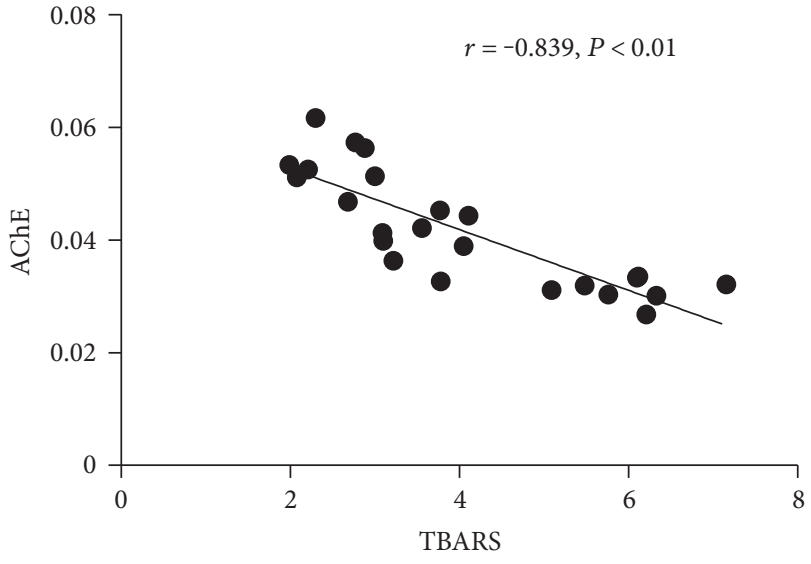

(a)

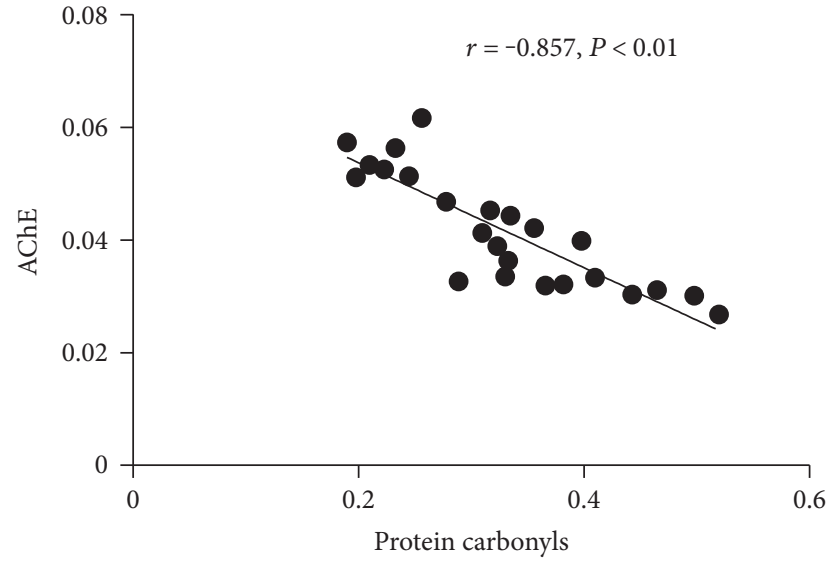

(b)

FIgURE 4: Correlation between AChE activity and TBARS (a) or protein carbonyls (b).

\section{Discussion}

Using low-grade and midgrade models of sepsis in rats, this study found that the AChE and CAT activities in the diaphragm decreased, while the contents of TBARS and protein carbonyls, the activity of MPO and SOD, and the SOD/CAT ratios increased at $24 \mathrm{~h}$ after CLP surgery. Furthermore, the above changes were much more significant in the midgrade septic group than those in the low-grade septic group. Strikingly, we found that AChE activity was significantly and negatively correlated with the levels of TBARS and protein carbonyls in rats.

In this study, low-grade and midgrade sepsis was established by ligating different caecum lengths, as described in previous studies [19]. The rats in the midgrade septic group had a higher mortality rate and developed more severe septic symptoms than those in the low-grade septic group. Moreover, TBARS and protein carbonyls, which represent the lipid and protein injury, respectively, in oxidative stress increased more in the midgrade septic group than in the low-grade septic group. These results illustrated that our sepsis models of different severity grades were successfully created.

AChE activity was found to be significantly and negatively correlated with the levels of TBARS and protein carbonyls, which indicated that oxidative stress may contribute to the decreased AChE activity during sepsis. It is believed that ROS plays an important role in the oxidative stress to cause cellular injury during sepsis. ROS in the diaphragm may primarily be derived from mitochondrial respiration chain impairment and infiltrating inflammatory cells $[24,25] . \mathrm{O}_{2}^{\bullet-}$ produced through the above two manners can be converted to $\mathrm{H}_{2} \mathrm{O}_{2}$ by SOD. $\mathrm{H}_{2} \mathrm{O}_{2}$ will be converted to harmless $\mathrm{H}_{2} \mathrm{O}$ and $\mathrm{O}_{2}$ through the action of CAT. Otherwise, it will allow neutrophils to oxidize chloride ions into $\mathrm{HOCl}$ through MPO [26]. SOD and CAT are two oxidative enzymes that play vital roles in eliminating ROS. Some research has revealed that SOD activity increased without a proportional increase in CAT activity during sepsis [27, 28]. In our study, the imbalance also existed, and it was much more serious in midgrade sepsis than in low-grade sepsis. The different modulation of SOD and CAT during sepsis may rise from some reasons below. SOD activity increased in the CLP surgery probably as a response to oxidative stress induced by sepsis. SOD activation during sepsis could be a compensatory response to the overproduction of mitochondrial $\mathrm{O}_{2}{ }^{\bullet-}$ so as to eliminate excessive $\mathrm{O}_{2}{ }^{\bullet-}[28]$. Additionally, the increase of interleukin-1 (IL-1), tumor necrosis factor (TNF), and lipopolysaccharide (LPS) during sepsis could also activate SOD $[29,30]$. Unlike SOD, the previous study demonstrated that excessive $\mathrm{O}_{2}{ }^{\bullet-}$ or 
$\mathrm{H}_{2} \mathrm{O}_{2}$ could oxidize CAT active site leading to enzymatic inactivation [31]. The imbalance between SOD and CAT may result in the accumulation of excessive $\mathrm{H}_{2} \mathrm{O}_{2}$ in the diaphragm [27, 32]. Additionally, our results indicated that MPO activity increased in both septic groups, and the activity increased higher in midgrade sepsis than in low-grade sepsis. MPO, synthesized and secreted by neutrophils, is a marker of inflammation and neutrophil infiltration in tissues [33]. As described above, increased MPO can further convert excessive $\mathrm{H}_{2} \mathrm{O}_{2}$ into $\mathrm{HOCl}$. Moreover, excessive $\mathrm{H}_{2} \mathrm{O}_{2}$ will react with iron to generate ${ }^{\bullet} \mathrm{OH}$ through Fenton chemistry [28].

Early in 1966, O'Malley et al. found that both $\mathrm{H}_{2} \mathrm{O}_{2}$ and peroxides could inhibit erythrocyte AChE [11]. A study from Danylovych reported that AChE of myometrium sarcolemma could be inhibited by $\mathrm{H}_{2} \mathrm{O}_{2}$ [12]. Accumulation of $\mathrm{H}_{2} \mathrm{O}_{2}$ in the $\mathrm{mM}$ range can decrease epidermal AChE expression and inhibit human recombinant AChE activity $[13,14]$. Additionally, it has been verified that ${ }^{\circ} \mathrm{OH}$ inhibits rat brain AChE and human recombinant AChE activity $[15,16]$. In addition, $\mathrm{HOCl}$ has been found to be a strong inhibitor of AChE [17]. A kinetic analysis using pure recombinant human AChE and a molecular modelling based on the established 3D structure of human AChE supported that ROS-mediated oxidation of Trp432, Trp435, and Met436 moves and disorients the active site His440 of AChE, leading to deactivation of the protein $[13,14]$. Moreover, TBARS can decrease membrane fluidity and decrease AChE activity through lipid-protein interactions [15]. These results provided a molecular basis through which oxidative stress inhibits AChE activity during sepsis.

However, our study has two limitations. First, we failed to observe the conformation change of AChE induced by ROS directly because of the limitations of the experimental methods. Second, we did not detect the quantity of AChE expression simultaneously, although recent data from another study in our laboratory revealed that such data may be another factor affecting the decrease of AChE activity (unpublished data).

\section{Conclusions}

We believe that there were at least two highlights in the present study. First, we found that AChE activity at the NMJ of diaphragm decreased more significantly during severe sepsis for the first time. Second, AChE activity at the NMJ of diaphragm is found to be significantly and negatively correlated with level of oxidative stress during sepsis.

In conclusion, our study verified that oxidative stress might be responsible for the decreased activity of the AChE at the NMJ in the diaphragm. Furthermore, the effects of antioxidant measurements on $\mathrm{AChE}$ activity during sepsis should be investigated in the future.

\section{Conflicts of Interest}

All authors declare that there is no conflict of interests regarding the publication of this paper.

\section{Authors' Contributions}

Hua Liu and Jin Wu contributed equally.

\section{Acknowledgments}

This study was supported by a grant from the National Natural Science Foundation of China (Grant no. 81171845).

\section{References}

[1] M. Singer, C. S. Deutschman, and C. W. Seymour, "The third international consensus definitions for sepsis and septic shock (sepsis-3)," JAMA, vol. 315, no. 8, pp. 801-810, 2016.

[2] A. Demoule, B. Jung, H. Prodanovic et al., "Diaphragm dysfunction on admission to ICU: prevalence, risk factors and prognostic impact - a prospective study," American Journal of Respiratory and Critical Care Medicine, vol. 88, no. 2, pp. 213-219, 2013.

[3] J. Wu, T. Jin, H. Wang, and S.-T. Li, "Sepsis strengthens antagonistic actions of neostigmine on rocuronium in a rat model of cecal ligation and puncture," Chinese Medical Journal, vol. 129, no. 12, pp. 1477-1482, 2016.

[4] R. C. Bone, C. J. Grodzin, and R. A. Balk, "Sepsis: a new hypothesis for pathogenesis of the disease process," Chest, vol. 112, no. 1, pp. 235-243, 1997.

[5] R. C. Bone, R. A. Balk, F. B. Cerra, R. P. Dellinger, A. M. Fein, and W. A. Knaus, "Definitions for sepsis and organ failure and guidelines for the use of innovative therapies in sepsis," Chest, vol. 101, no. 6, pp. 1644-1655, 1992.

[6] D. Rittirsch, M. A. Flierl, and P. A. Ward, "Harmful molecular mechanisms in sepsis," Nature Reviews Immunology, vol. 8, no. 10, pp. 776-787, 2008.

[7] J. Duran-Bedolla, M. A. Montes de Oca-Sandoval, and V. Saldaña-Navor, "Sepsis, mitochondrial failure and multiple organ dysfunction," Clinical and Investigative Medicine, vol. 37, no. 2, pp. E58-E69, 2014.

[8] M. P. Fink, "Role of reactive oxygen and nitrogen species in acute respiratory distress syndrome," Current Opinion in Critical Care, vol. 8, no. 1, pp. 6-11, 2002.

[9] H. Bayir, "Reactive oxygen species," Critical Care Medicine, vol. 33, no. 12, pp. S498-S501, 2005.

[10] C. A. Prauchner, "Oxidative stress in sepsis: pathophysiological implications justifying antioxidant co-therapy," Burns, vol. 43, no. 3, pp. 471-485, 2017.

[11] B. W. O’Malley, C. E. Mengel, and W. D. Meriwether, "Inhibition of erythrocyte acetylcholinesterase by peroxides," Biochemistry, vol. 5, no. 1, pp. 40-44, 1966.

[12] D. IuV, "Hydrogen peroxide inhibits acetylcholinesterase of myometrium sarcolemma," UkrBiokhim Zh, vol. 81, no. 4, pp. 32-38, 2009.

[13] K. U. Schallreuter, S. M. Elwary, and N. C. Gibbons, “Activation/deactivation of acetylcholinesterase by $\mathrm{H} 2 \mathrm{O} 2$ : more evidence for oxidative stress in vitiligo," Biochemical and Biophysical Research, vol. 315, no. 2, pp. 502-508, 2004.

[14] K. U. Schallreuter and S. Elwary, "Hydrogen peroxide regulates the cholinergic signal in a concentration dependent manner," Life Sciences, vol. 80, no. 24-25, pp. 2221-2226, 2007.

[15] S. Tsakiris, P. Angelogianni, K. H. Schulpis, and J. C. Stavridis, "Protective effect of $\mathrm{L}$ phenylalanine on rat brain 
acetylcholinesterase inhibition induced by free radicals," Clinical Biochemistry, vol. 33, no. 2, pp. 103-106, 2000.

[16] A. Méndez-Garrido, M. Hernández-Rodríguez, R. ZamoranoUlloa et al., "In vitro effect of $\mathrm{H} 2 \mathrm{O} 2$, some transition metals and hydroxyl radical produced via Fenton and Fenton-like reactions, on the catalytic activity of $\mathrm{AChE}$ and the hydrolysis of ACh," Neurochemical Research, vol. 39, no. 11, pp. 20932104, 2014.

[17] G. J. den Hartog, E. Vegt, and W. J. van der Vijgh, "Hypochlorous acid is a potent inhibitor of acetylcholinesterase," Toxicology and Applied Pharmacology, vol. 181, no. 3, pp. 228-232, 2002.

[18] D. Rittirsch, M. S. Huber-Lang, M. A. Flierl, and P. AWard, "Immunodesign of experimental sepsis by cecal ligation and puncture," Nature Protocols, vol. 4, no. 1, pp. 31-36, 2009.

[19] S. Ruiz, F. Vardon-Bounes, V. Merlet-Dupuy, J.-M. Conil, M. Buléon, and O. Fourcade, "Sepsis modeling in mice: ligation length is a major severity factor in cecal ligation and puncture," Intensive Care Medicine Experimental, vol. 4, no. 1, p. 22, 2016

[20] M. J. Karnovsky and L. Roots, "A "direct-coloring” thiocholine method for cholinesterases," The Journal of Histochemistry and Cytochemistry, vol. 12, no. 3, pp. 219-221, 1964.

[21] S. Ma, S. Xu, B. Liu et al., "Long-term treatment of 1-3-nbutylphthalide attenuated neurodegenerative changes in aged rats," Naunyn-Schmiedeberg's Archives of Pharmacology, vol. 379, no. 6, pp. 565-574, 2009.

[22] H. H. Draper and M. Hadley, "Malondialdehyde determination as index of lipid peroxidation," Methods in Enzymology, vol. 186, pp. 421-431, 1990.

[23] R. L. Levine, D. Garland, and C. N. Oliver, "Determination of carbonyl content in oxidatively modified proteins," Methods in Enzymology, vol. 186, pp. 464-478, 1990.

[24] D. E. Taylor, A. J. Ghio, and C. A. Piantadosi, "Reactive oxygen species produced by liver mitochondria of rats in sepsis," Archives of Biochemistry and Biophysics, vol. 316, no. 1, pp. 70-76, 1995.

[25] Y. V. Lerman and M. Kim, "Neutrophil migration under normal and sepsis conditions," Cardiovascular \& Hematological Disorders Drug Targets, vol. 15, no. 1, pp. 19-28, 2015.

[26] J. P. Gaut, G. C. Yeh, and H. D. Tran, "Neutrophils employ the myeloperoxidase system to generate antimicrobial brominating and chlorinating oxidants during sepsis," Proceedings of the National Academy of Sciences of the United States of America, vol. 98, no. 21, pp. 11961-11966, 2001.

[27] C. Ritter, M. E. Andrades, A. Reinke, S. Menna-Barreto, J. C. Moreira, and F. Dal-Pizzol, "Treatment with N-acetylcysteine plus deferoxamine protects rats against oxidative stress and improves survival in sepsis," Critical Care Medicine, vol. 32, no. 2, pp. 342-349, 2004.

[28] C. Ritter, M. Andrades, and M. L. Frota Júnior, "Oxidative parameters and mortality in sepsis induced by cecal ligation and perforation," Intensive Care Medicine, vol. 29, pp. 17821789, 2003.

[29] A. Masuda, D. L. Longo, and Y. Kobayashi, "Induction of mitochondrial manganese superoxide dismutase by interleukin 1," The FASEB Journal, vol. 2, pp. 3087-3091, 1988.

[30] G. A. Visner, W. C. Douglas, J. M. Wilson, I. A. Burr, and H. S. Nick, "Regulation of manganese superoxide dismutase by lipopolysaccaride, interleukin-1, and tumor necrosis factor," The Journal of Biological Chemistry, vol. 265, pp. 2856-2286, 1990.
[31] T. G. Gantchev and J. E. van Lier, "Catalase inactivation following photosensitization with tetrasulfonated metallophthalocyanines," Photochemistry and Photobiology, vol. 62, pp. 123-134, 1995.

[32] C. A. Prauchner, S. Prestes Ade, and J. B. da Rocha, "Effects of diphenyl diselenide on oxidative stress induced by sepsis in rats," Pathology, Research and Practice, vol. 207, pp. 554558, 2011.

[33] M. Faith, A. Sukumaran, A. B. Pulimood, and M. Jacob, "How reliable an indicator of inflammation is myeloperoxidase activity?," Clinica Chimica Acta, vol. 396, pp. 23-25, 2008. 


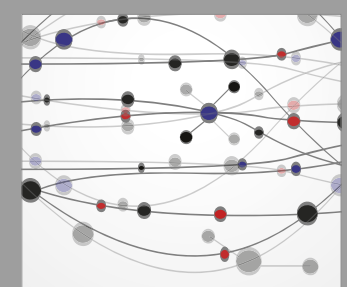

The Scientific World Journal
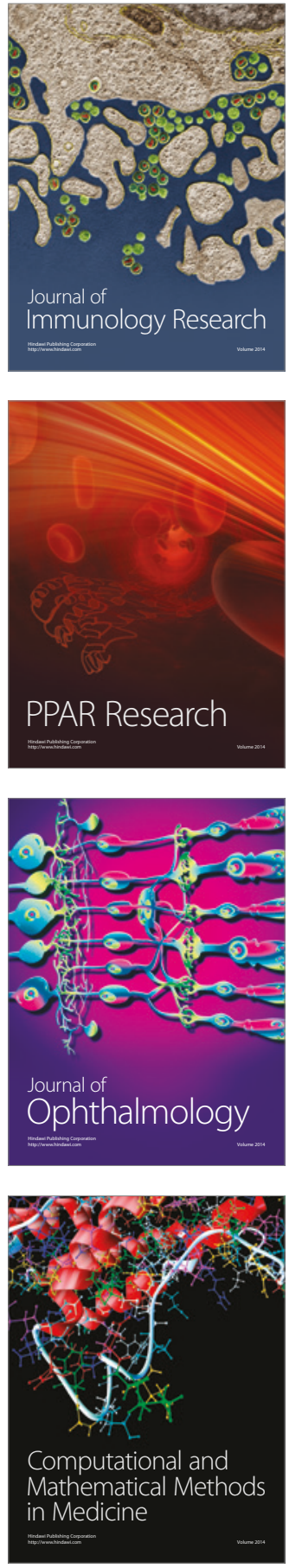

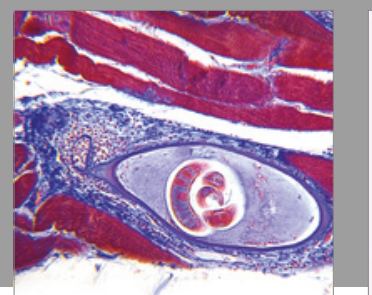

Gastroenterology Research and Practice
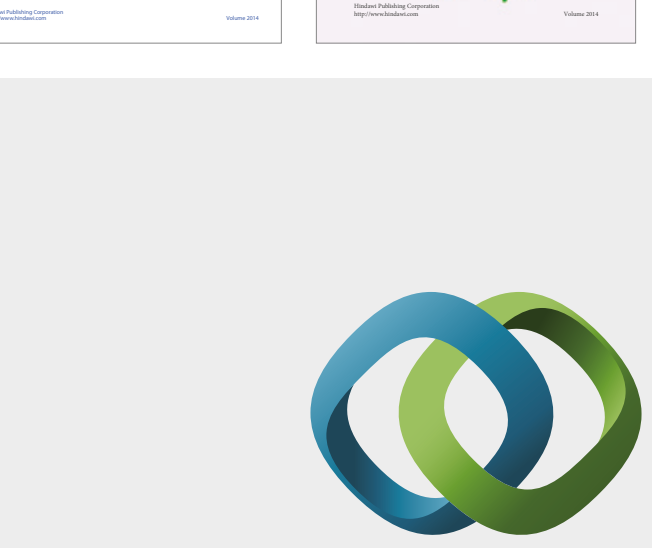

\section{Hindawi}

Submit your manuscripts at

https://www.hindawi.com
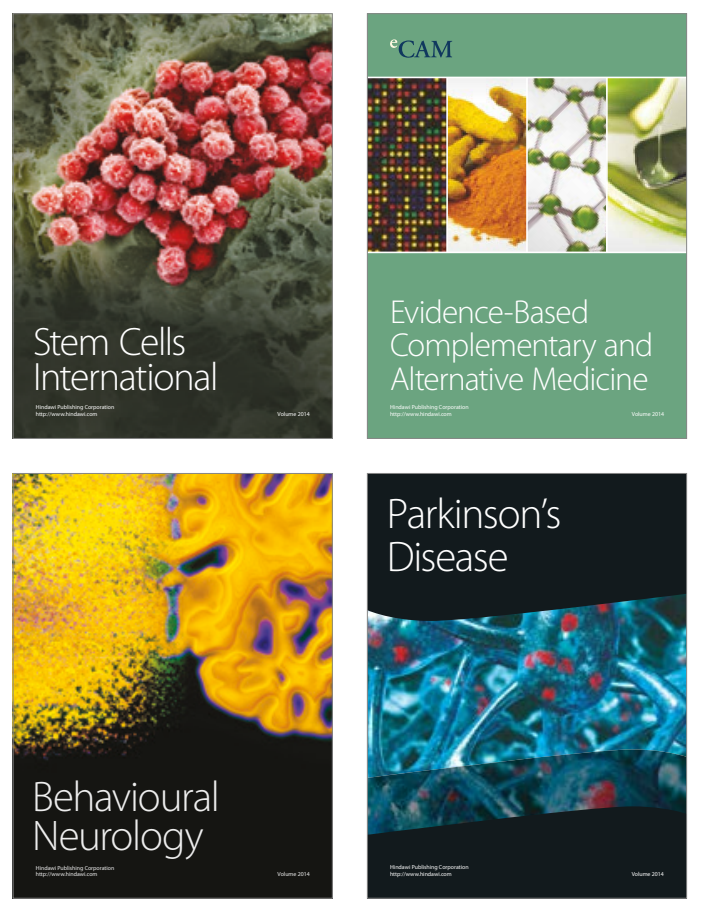
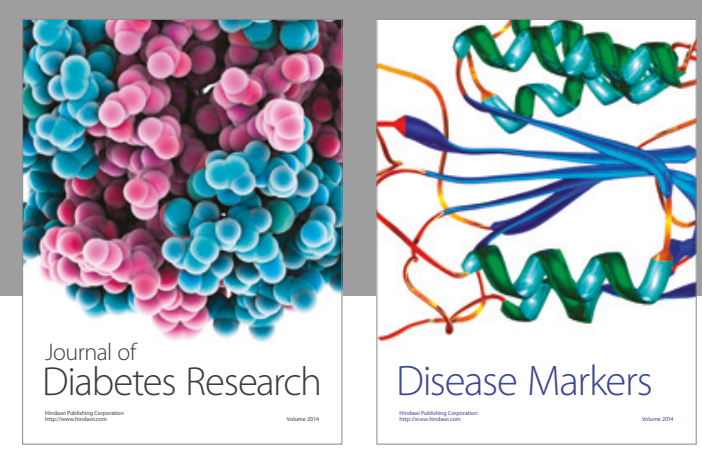

Disease Markers
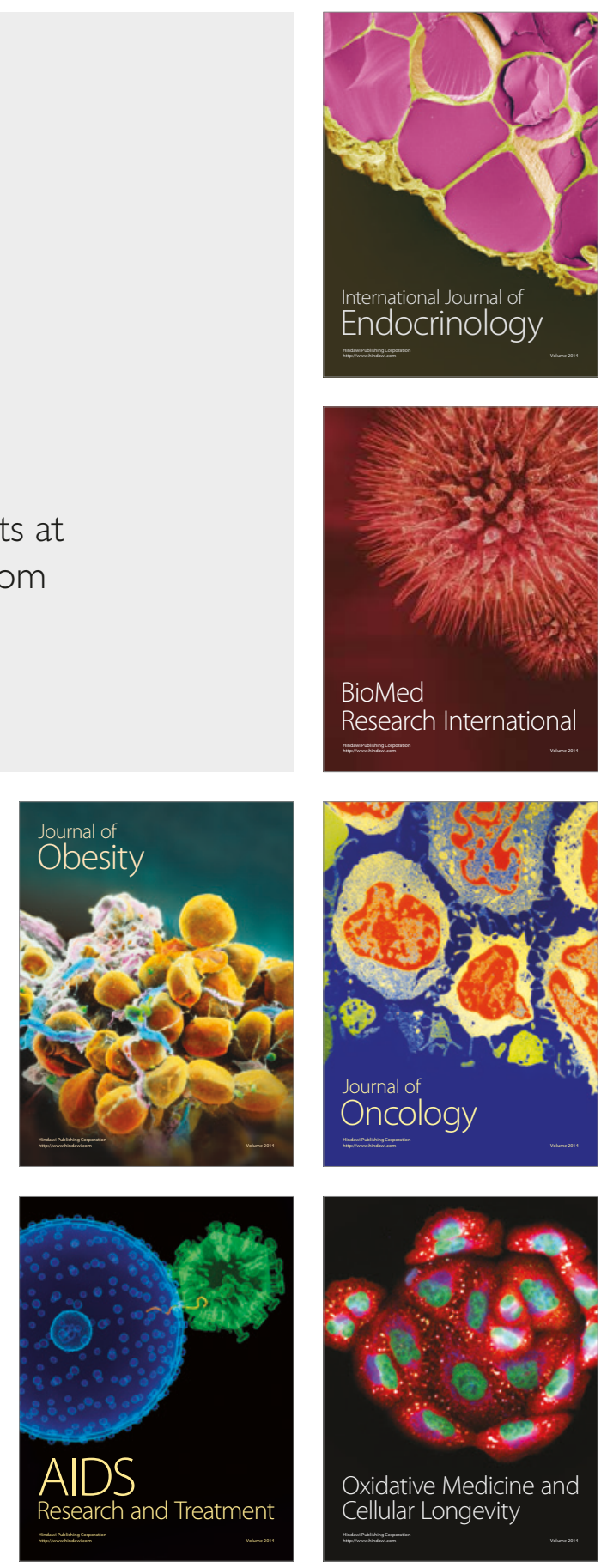